\title{
COVID-19 mRNA Vaccine Induced Troponinemia - Is the Vaccine a Cardiac Stressor?
}

\section{Sameer Acharya, $M D^{1^{*}}$ (D) Malcolm Brand, MD, FACC ${ }^{2}$, Janette Lee, $M D^{1}$, Douglas MacQueen, $M D^{3}$, Angela Arbach, $M D^{1}$ and Samjhauta Bhattarai, $M B B S^{4}$}

${ }^{1}$ Department of Internal Medicine, Cayuga Medical Center, New York, USA

${ }^{2}$ Department of Cardiology, Cayuga Medical Center, New York, USA

${ }^{3}$ Department of Infectious Diseases, Cayuga Medical Center, New York, USA

${ }^{4}$ Department of Internal Medicine, B and B Hospital, Kathmandu, Nepal

*Corresponding author: Sameer Acharya, MD, Department of Internal Medicine, Cayuga Medical Center, 101 Dates Drive, Ithaca, New York, 14850, USA, Tel: 332-201-4635

\begin{abstract}
There have been emerging concerns of myocarditis with Covid-19 vaccines recently which could be from inflammatory or immunogenic response to vaccine components or something else which is yet to be understood. The most common side effects of the vaccine noted in phase three trials were local reactions and few self-remitting systemic reactions. But we cannot overlook the emerging cardiac manifestations associated with COVID-19 vaccine. Here, we report a young healthy male presenting with atypical chest pain found with troponinemia most likely from myopericarditis, three days after his second dose of Pfizer-BNT162b2 vaccine. He was placed on telemetry monitoring and was treated with Aspirin $81 \mathrm{mg}$, Colchicine $0.6 \mathrm{mg}$ and Ibuprofen. Given his normal body mass index (BMI), renal function, lipids, d-Dimer, transthoracic echocardiogram (TTE), computed tomography angiography (CTA) chest and non-specific findings in electrocardiogram (EKG), and the close temporal relationship of an inflammatory response to his second $\mathrm{CO}$ VID-19 vaccination dose, we suspect that his cardiac pathology was a result of his response to vaccination and not to underlying atherosclerotic or embolic disease.
\end{abstract}

\section{Keywords}

Covid-19 vaccine, Cardiac risk, Myocarditis, Myopericarditis, Troponinemia, Chest pain

\section{Abbreviations}

BMI: Body Mass Index; COVID-19: Coronavirus Disease (COVID-19); CTA: Computed Tomography Angiography; EKG: Electrocardiogram; HIV: Human Immune Deficiency Virus; LV: Left Ventricle; mRNA: Messenger Ribonucleic Acid; TTE: Transthoracic Echocardiography

\section{Introduction}

Coronavirus disease (COVID-19) has challenged the world since March 11, 2020 [1], affecting hundreds of millions of people globally [2]. The advent of an effective vaccine offered hope for countering the pandemic and normalization of lives. The two-dose regimen of 30 micrograms of the messenger RNA (mRNA)based vaccine BNT-162b2 using lipid nanoparticles to facilitate the transport of mRNA into cells from PfizerBioNTech is known to be safe and $95 \%$ effective from phase three trials [3]. The most common side effects of the vaccine noted in phase three trials were local reactions (pain, erythema, and swelling) and systemic reactions like fever, malaise, and myalgia, which usually resolved rapidly [3,4]. Overall, systemic adverse events were noted more frequently in the younger age group as compared to the older group [3]. Here, we report a case of a healthy young man who presented with severe chest pain and markedly elevated troponins after getting the second dose of the BNT162b2 vaccine, with no evidence of established coronary artery disease.

\section{Case Description}

A healthy 18-year-old male, who received his second dose of Pfizer-BNT162b2 vaccine three days prior to presentation, came to the emergency department with subacute, intermittent, left-sided chest pain,

Citation: Acharya S, Brand M, Lee J, MacQueen D, Arbach A, et al. (2021) COVID-19 mRNA Vaccine Induced Troponinemia - Is the Vaccine a Cardiac Stressor?. Int J Clin Cardiol 8:232. doi.org/10.23937/23782951/1410232

Accepted: July 01, 2021: Published: July 03, 2021

Copyright: (C) 2021 Acharya S, et al. This is an open-access article distributed under the terms of the Creative Commons Attribution License, which permits unrestricted use, distribution, and reproduction in any medium, provided the original author and source are credited. 
which began approximately two days after receiving the vaccine. He reported a temperature of $38.5^{\circ} \mathrm{C}$ with nausea, vomiting, and malaise shortly after vaccination, and subsequently the abrupt onset of left-sided chest pain radiating to the neck and left upper arm that was non-exertional, non-pleuritic, and non-positional in nature. This episode of chest pain lasted for an hour, and after remitting for approximately 10 hours, the second episode of chest pain followed and persisted for a couple of hours; both episodes occurred at rest and resolved spontaneously. He did not have associated dyspnea or cough. His vital signs on presentation were: blood pressure 144/76 mmHg, pulse 93 beats per minute, respirations 16 breaths per minute, oxygen saturation $96 \%$ on room air, temperature $37.6{ }^{\circ} \mathrm{C}$. His BMI was $22.8 \mathrm{~kg} / \mathrm{m}^{2}$. The cardiovascular and other systemic examination was unremarkable. The patient had vitamin D deficiency with no history of hypertension, hyperlipidemia, diabetes, congenital heart disease, autoimmune disease, or endocrine disorders. He was taking vitamin D supplements. Initially, atypical chest pain occurring at rest had put our patient at high suspicion for angina, acute coronary syndrome, coronary artery spasm, myocarditis, myopericarditis, pericarditis, or pericardial effusion. Pulmonary embolism and gastroesophageal reflux disease were also in the differential but lower on the list.

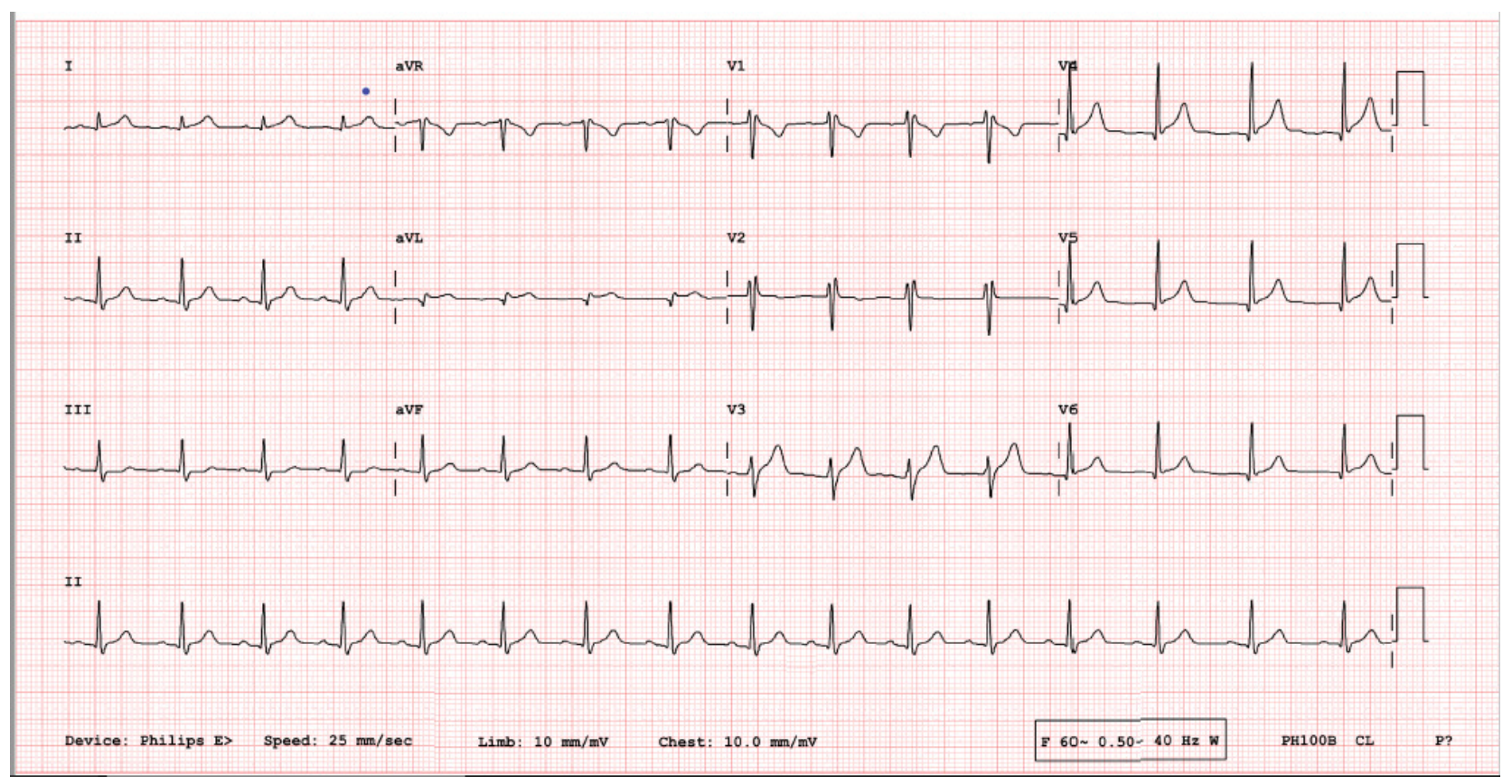

Figure 1: Initial 12 lead EKG showing non-specific ST elevations in anterior and lateral leads.

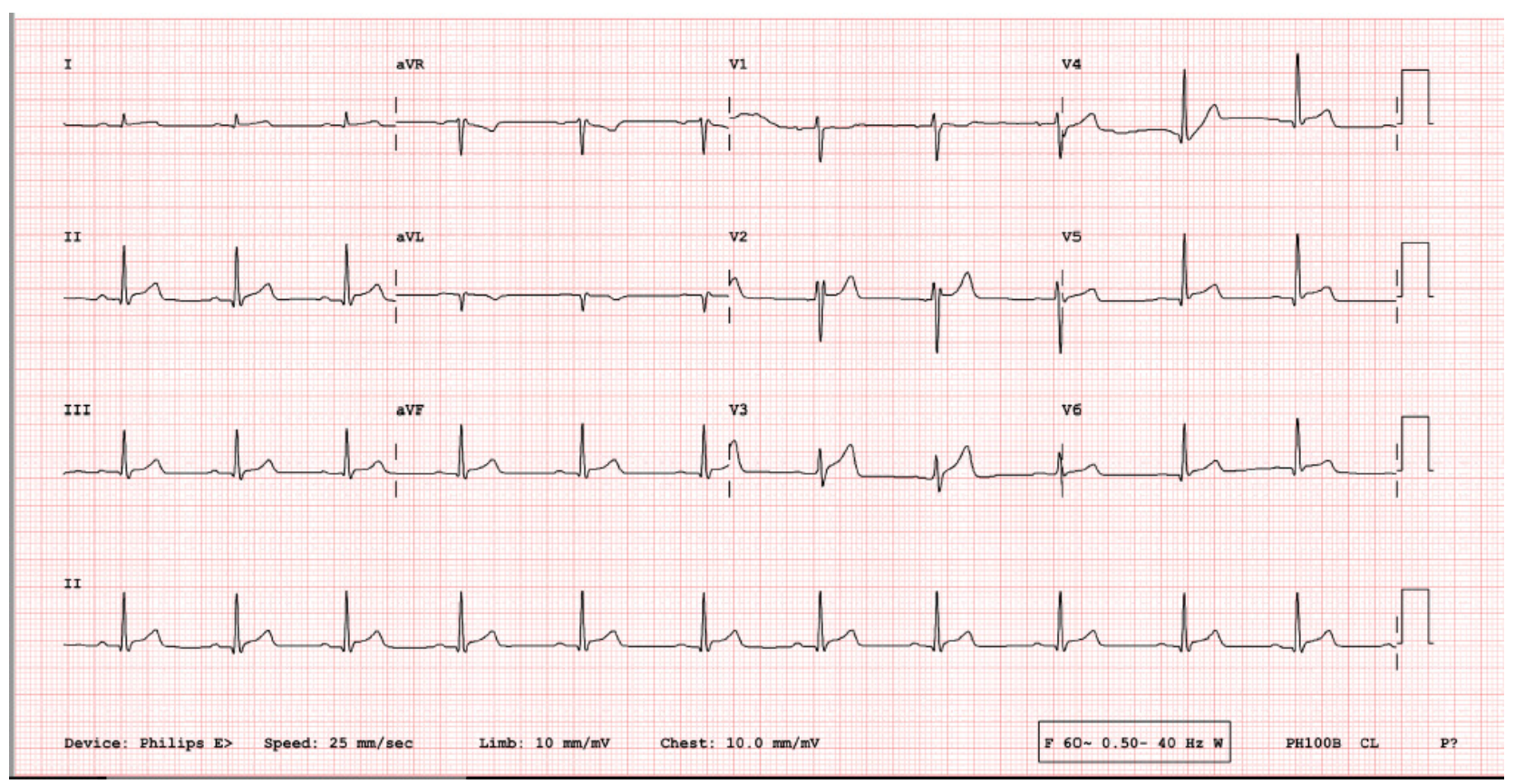

Figure 2: Repeat 12 lead EKG showing non-specific diffuse ST elevations. 
Complete blood count, comprehensive metabolic panel, lipid panel, and d-dimer were normal. Troponin I was $5.31 \mathrm{ng} / \mathrm{ml}$ (normal range $<0.03 \mathrm{ng} / \mathrm{ml}$ ), which increased to $5.68 \mathrm{ng} / \mathrm{ml}$ and then decreased to 3.06 $\mathrm{ng} / \mathrm{ml}$ after 28 hours. His total creatine kinase was $708 \mathrm{U} / \mathrm{l}, \mathrm{CK}-\mathrm{MB} 38 \mathrm{ng} / \mathrm{ml}$, C-reactive protein $21.8 \mathrm{mg} / \mathrm{l}$ and erythrocyte sedimentation rate was 15 . COVID-19 polymerase chain reaction of the nasopharyngeal swab was negative. Baseline 12-lead EKG showed non-specific ST elevations in anterior and lateral leads (Figure 1) which were progressed to non-specific diffuse ST elevation on repeat EKG (Figure 2).

CTA of the chest showed normal lungs, no calcium deposits in the aorta or coronary arteries, and no evidence of pericardial effusion. TTE showed normal systolic and diastolic function, left ventricular (LV) ejection fraction of $55-60 \%$, normal chamber size, no regional wall motion abnormalities, and no evidence of pericardial effusion (Figure 3, Figure 4 and Figure 5). His urine toxicology was negative for toxins, and serology for hepatitis A, B, C, Cytomegalovirus IgG and IgM, Epstein Barr, Enterovirus, HIV, and Influenza A and B were negative.

He was placed on telemetry monitoring and was treated with Aspirin $81 \mathrm{mg}$, Colchicine $0.6 \mathrm{mg}$, and Ibuprofen. He never developed hypoxia or arrhythmia during the entire hospital stay. He was symptom-free with down-trending troponins and was discharged after two days of hospitalization with three months' dose of
Aspirin $81 \mathrm{mg}$ and Colchicine $0.6 \mathrm{mg}$ daily and Ibuprofen as needed.

\section{Discussion}

Myocarditis is an inflammatory condition of the heart muscle due to infectious causes, inflammatory conditions, autoimmune disorders, or toxins whose severity varies from self-resolving illnesses to lifethreatening events like sudden cardiac death, if not treated in time. The EKG findings in myopericarditis may include non-specific ST changes, $Q$ waves, single atrial or ventricular ectopic beats, complex ventricular arrhythmias or rarely atrial tachycardia or atrial fibrillation [5]. TTE evaluation in myocarditis range from normal findings to global or regional wall motion abnormalities or vast changes in LV dimensions and geometry along with co-existent pericardial involvement, silent intracardiac thrombi or valvular dysfunctions [5]. But, still TTE is the crucial method of detecting suboptimal ventricular function in suspected myocarditis [5]. There is a risk of the immunogenic or inflammatory response to vaccine lipid nanoparticles or mRNA components [6], leading to inflammation of cardiac muscle or pericardium, and this scenario has been a topic of much interest and investigation [7]. Although cases of myocardial infarction due to other COVID-19 vaccines have been reported infrequently [8], cardiac events with BNT-162b2 have been very uncommon to date [7]. Moreover, severe adverse events during the phase 3 trials of the Pfizer-BNT162b2

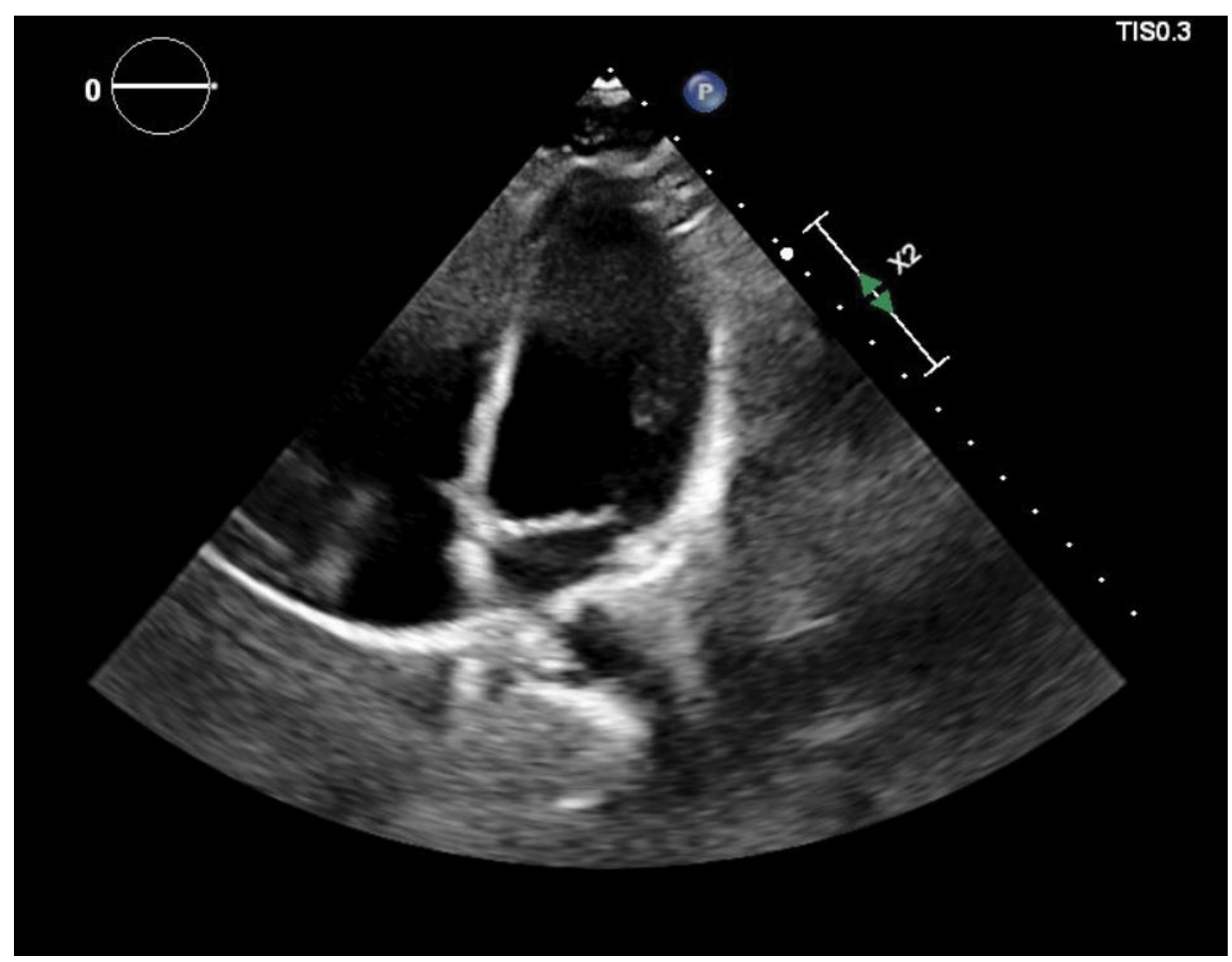

Figure 3: Four chamber view on TTE showing no pericardial effusion and normal chamber size. 


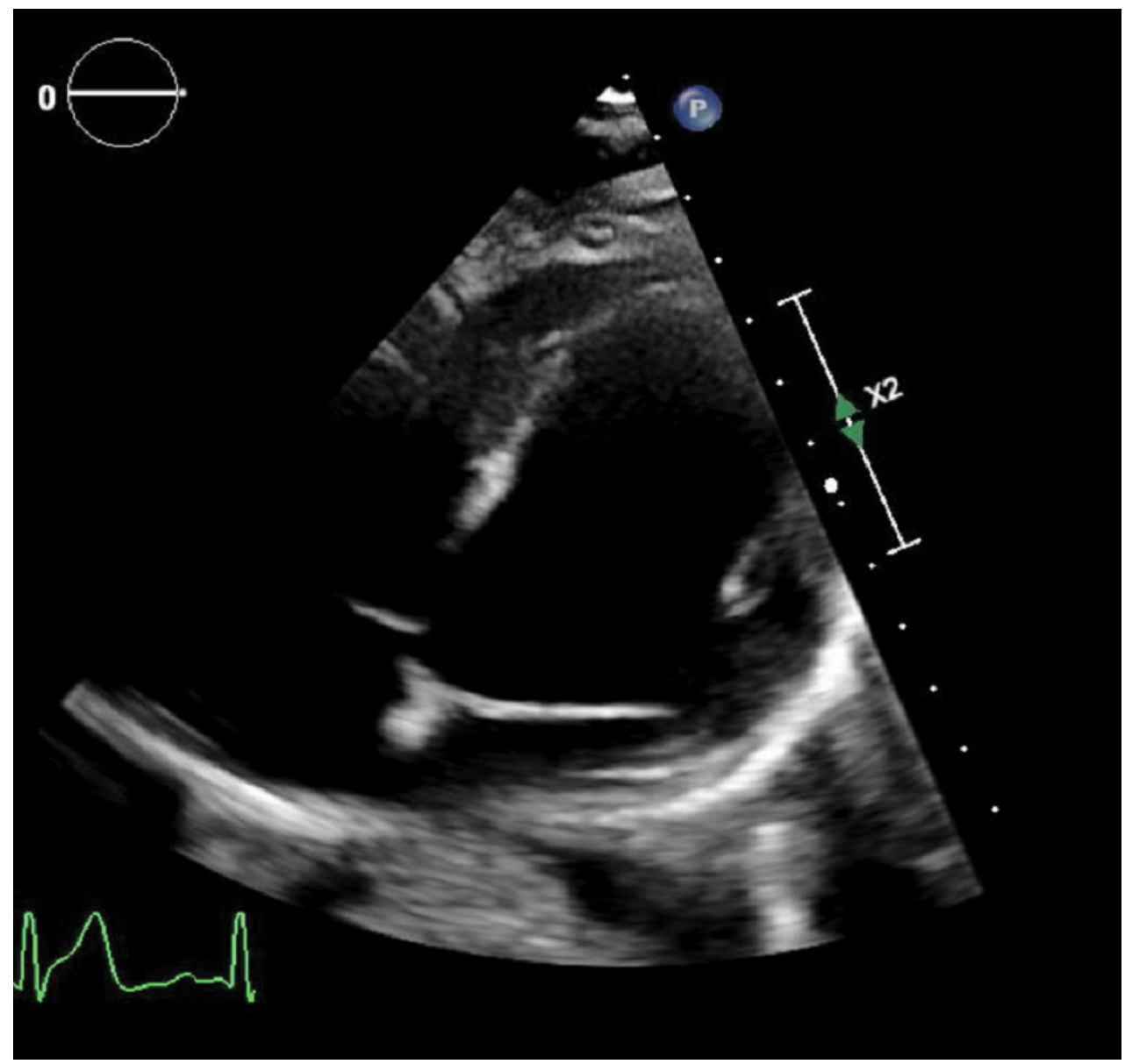

Figure 4: Modified right ventricular focus on TTE showing normal pericardial space.

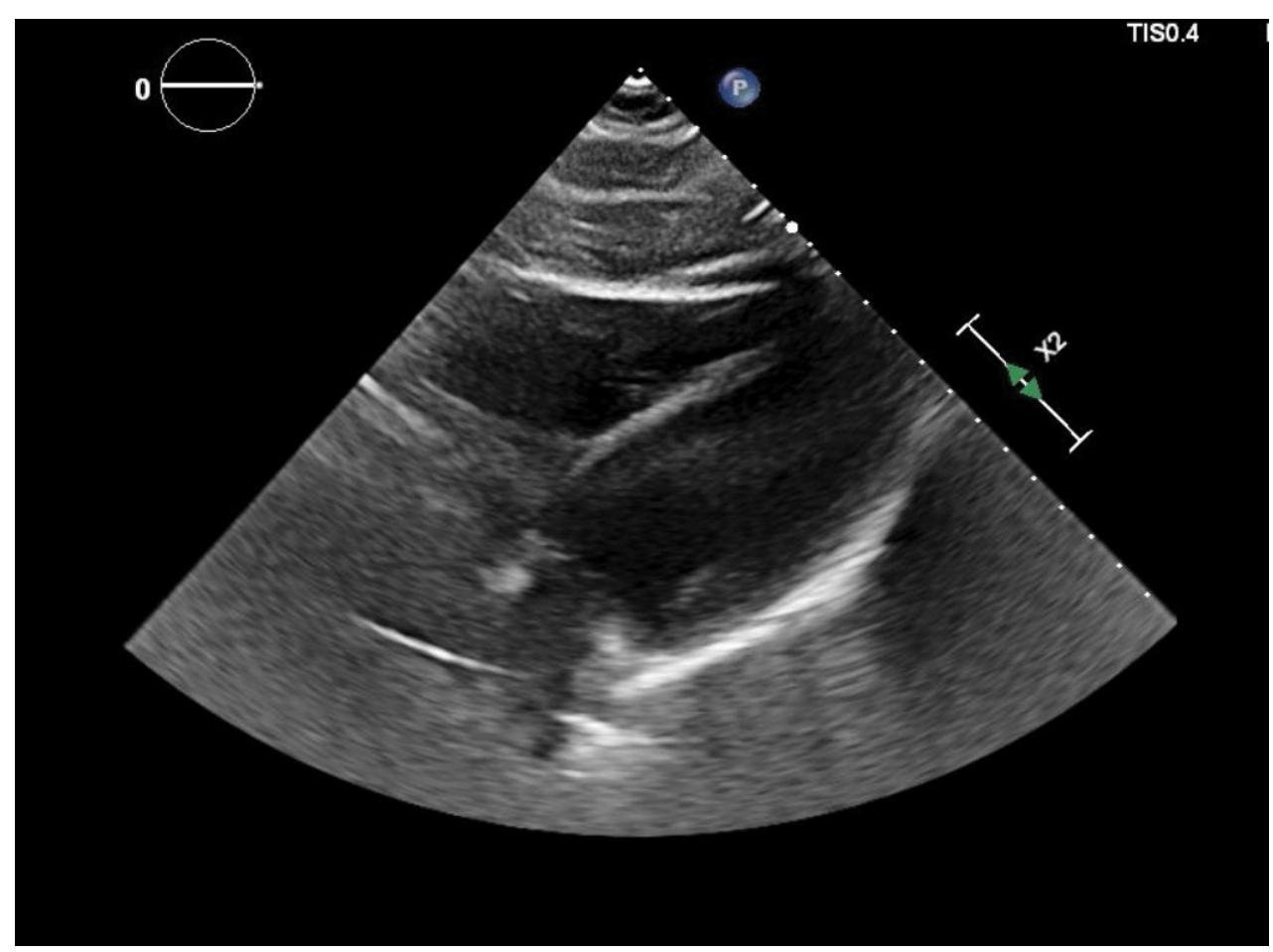

Figure 5: Subcostal view on TTE showing normal chamber size.

vaccine were rare, including shoulder injury related to vaccine administration, right axillary lymphadenopathy, paroxysmal ventricular arrhythmia, and right leg paresthesia. Two deaths were reported among BNT-
$162 \mathrm{~b} 2$ recipients (one from arteriosclerosis, one from cardiac arrest). As the vaccine eligibility extends to broader segments of the population, new adverse reactions are being reported $[6,9]$, anaphylaxis reactions 
being the most common thusfar $[10,11]$. There have been the global concerns about rare association of COVID-19 mRNA vaccine with myocarditis or myopericarditis especially among teens and young adults which is still under investigation [12]. Given our young patient's normal BMI, renal function, lipids, d-Dimer, TTE, CTA chest and non-specific findings in EKG, and the close temporal relationship of an inflammatory response to his second COVID-19 vaccination dose, we suspect that his cardiac pathology was a result of his response to vaccination and not to underlying atherosclerotic or embolic disease. Although cardiac magnetic resonance imaging might have helped to demonstrate an explicit diagnosis of myocarditis or myopericarditis, we made the clinical decision to not pursue this test based upon his improving clinical status and cardiac biomarkers. He is being regularly followed up in the cardiology clinic and has been completely asymptomatic.

\section{Conclusion}

To the best of our knowledge, this is one of the very few cases of elevated troponin levels likely due to myocarditis or myopericarditis, associated with the BNT-162b2 vaccine reported thus far. In all probability, the high troponin level was a result of an immunogenic or inflammatory response to the mRNA vaccine, leading due to myopericarditis or myocarditis, given the absence of other clear causes. The 12 lead EKG with non-specific diffuse ST elevations in the setting of acute chest pain and elevated cardiac biomarkers led us to the diagnosis of myopericarditis which was reinforced by the patient's quick response to the anti-inflammatory therapy. Cardiac MRI would have shown myocardial edematous changes but it was not warranted due to his improving clinical status. From this case, the authors want to provide a rationale for close evaluation of anyone with chest symptoms after receiving the COVID-19 vaccine. Since there have been emerging concerns of cardiac involvement in young patients without atherosclerotic cardiovascular disease after the COVID-19 vaccine, further studies will be needed to define a link between the vaccine, symptoms, and elevated cardiac biomarkers. While it is premature to assume causation of coronary events from the vaccine, the authors of this case want to acknowledge that, while we advocate for universal vaccine coverage, we should always be vigilant about unexpected adverse events that could pose diagnostic dilemmas and risk to our patients.

\section{Funding}

The author(s) have disclosed that there was no funding required for the preparation of this paper.

\section{Statement of Equal Authors' Contribution}

All authors made equal contributions in designing the case report directed its implementation, including quality assurance and control. All authors interpreted the data and critically reviewed and revised the final version of the manuscript. All authors read and approved the final version of the manuscript.

\section{Acknowledgements}

The authors would like to thank the Cayuga Heart Institute of Cayuga Medical Associates, Cayuga Medical Center staff, patient and the family members.

\section{Conflict of Interest}

None.

\section{Disclosures}

The authors have reported that they have no relationships relevant to the contents of this paper to disclose.

\section{References}

1. WHO (2021) WHO Director-General's opening remarks at the media briefing on COVID-19-11 March 2020.

2. Johns Hopkins University (2021) Johns Hopkins University Coronavirus Resource Center. COVID-19 dashboard by the Center for Systems Science and Engineering (CSSE) at Johns Hopkins University.

3. Polack FP, Thomas SJ, Kitchin N, Absalon J, Gurtman A, et al. (2020) Safety and efficacy of the BNT162b2 mRNA Covid-19 Vaccine. N Engl J Med 383: 2603-2615.

4. CDC (2021) Pfizer-BioNTech COVID-19 vaccine overview and safety.

5. Cooper LT (2021) Clinical manifestations and diagnosis of myocarditis in adults.

6. Remmel A (2021) COVID vaccines and safety: What the research says. Nature 590: 538-540.

7. Mandavilli A (2021) C.D.C. Is Investigating a Heart Problem in a Few Young Vaccine Recipients. The New York Times.

8. Boivin Z, Martin J (2021) Untimely myocardial infarction or COVID-19 vaccine side effect. Cureus 13: e13651.

9. Carli G, Nichele I, Ruggeri M, Barra S, Tosetto A (2021) Deep vein thrombosis (DVT) occurring shortly after the second dose of mRNA SARS-CoV-2 vaccine. Intern Emerg Med 16: 803-804.

10. Garvey LH, Nasser S (2020) Anaphylaxis to the first COVID-19 vaccine: Is polyethylene glycol (PEG) the culprit? Br J Anaesth 126: e106-e108.

11. Rodriguez-Nava G, Egoryan G, Trelles-Garcia DP, YanezBello MA, Murguia-Fuentes R (2021) Disproportionality analysis of anaphylactic reactions after vaccination with messenger RNA coronavirus disease 2019 vaccines in the United States. Ann Allergy Asthma Immunol 127: 139-140.

12. AHA (2021) CDC investigating rare myocarditis in teens, young adults; COVID-19 vaccine still advised for all who are eligible. 